\title{
The Evolution of the Strategies of Innovation Cooperation in Scale-Free Network
}

\author{
Bing Zhu ${ }^{1,2}$ and Wenping Wang ${ }^{2}$ \\ ${ }^{1}$ School of Economics and Management, Anhui Normal University, Wuhu, Anhui 241002, China \\ ${ }^{2}$ School of Economics and Management, Southeast University, Nanjing, Jiangsu 211189, China
}

Correspondence should be addressed to Bing Zhu; zb_bing@sina.com

Received 14 January 2014; Accepted 19 February 2014; Published 31 March 2014

Academic Editor: Jinde Cao

Copyright (C) 2014 B. Zhu and W. Wang. This is an open access article distributed under the Creative Commons Attribution License, which permits unrestricted use, distribution, and reproduction in any medium, provided the original work is properly cited.

One of the important research works is corporate partnering and reliance on various forms of external collaboration. Although many researchers have focused on the knowledge-based work to help firms collaborate and share their knowledge, few have comprehensively considered the structure of network and cooperative strategies. Our paper differentiates three situations of innovation, and simulates the actions of cooperation between firms with different life cycles in industry cluster with the scale-free network structure. The simulation results show that (a) when the industry cluster develops, firms' absorbability also is growing, but the behaviors of cooperation do not always increase as firms' absorbability grows. Sometimes they exhibit inverted U-shape, Ushape, or liner trend. (b) Firms will cooperate to share their information or knowledge automatically if the payoff of the innovation is bigger. (c) The incentive policy does encourage the cooperation between the firms, but the incentive policy about compensation with each cooperation action is more effective than with the periodic fixed incentive.

\section{Introduction}

There has been unprecedented growth in corporate partnering and reliance on various forms of external collaboration [1-3]. Strategic alliances are increasingly necessary to support innovative activities [4]. These various types of interfirm alliances take on many forms, ranging from $R \& D$ partnerships to equity joint ventures to collaborative manufacturing to complex comarketing arrangements [5]. Numerous studies find that geographical proximity tends to facilitate innovation [6-8]. The interfirm cooperation can be extremely effective in increasing the circulation of tacit knowledge and in creating possibilities for a firm to acquire knowledge outside its boundaries and also facilitates development of capabilities within target organizations to manage the process of innovation [9].

Industrial clusters are geographic concentrations of interconnected companies and organizations which cooperate but may also compete with each other. The studies on clusters date back to Marshall's external economies. Following Marshall, Arrow [10] analyzed the nature of invention and technological advance and Romer [11] further developed specialization.
In 1990, Harvard Business School Professor Michael Porter examined industrial clusters from the perspective of business strategy and discussed the national and regional competitiveness. In consequence, cluster economies became a target for public policy [12]. A strand of studies has recognized the importance of the dynamics of clusters, while many earlier studies concentrated on the participants and advantages of a cluster. And also studies have emphasized the importance of networks of innovators and spatial proximity to explain the phenomenon of clustering $[13,14]$.

Recent studies confirmed the contribution of inventors' mobility to knowledge transfer within geographical clusters and began to study the role of social networks. The structure of network may emerge many forms with the development of the industry cluster. Small-world model is often seen as the major contending models for ubiquitous type of network. Another model is the scale-free network. Barabási and Albert [15] proposed scale-free network models as congruent to some of the more ubiquitous forms of centralization in networks. Many applications of the models have been made, including interfirm alliance formation and joint ventures and emergence of industry clusters, organizational learning, 
knowledge evolvement, dynamic capabilities' extending, and innovation mechanism [16-18].

With the development of industrial cluster, some large enterprise groups emerge, and many small and mediumsized enterprises are small. They prefer to link with the large enterprise access to information, resources, markets, and technologies. With the network evolution, the network structure of industrial cluster is the scale-free network. And it emerges in many places in China. So study issues in the scale-free network will be meaningful. Now there are some works exploring various aspects on the evolution in complex networks; see [19-21]. The existence of cooperationpromoting feedback mechanisms that are rooted deep into the interaction structure is indisputably accepted.

However, there is little explicit examination of the cooperation strategies in cluster network. As to the research of cooperative strategy, many scientists from different fields often resort to game theory, especially that of the Prisoner's Dilemma Game (PDG) with an evolutionary context [2225], as a common mathematical framework for investigating the dilemma of cooperation between individuals. And many efforts have been made to understand the conditions that can enable cooperative behavior to emerge and persist [2628]. It is found that several strategies with memory, such as "tit-for-tat" [29] and "win stay and lose shift" [30], can enhance the cooperation. But previous works mainly ignore connecting the heterogeneity of firms' capability, strategy, and the structure of innovation network.

So the purpose of this paper will consider both the structure of innovation network and the heterogeneity of firms' capability and includes examining how the different strategies evolve and what is the appropriate various incentives police under different situations of innovation in the cluster.

\section{The Model}

We begin with a schematic description of the model, before giving the technical details of the parameterization of the network architecture and the situations of innovation, agents' capability distribution, and incentive policies. We are interested in the effects of the cooperation strategies evolvement and the effects of incentive policies.

2.1. Basic Payoff Matrix. For the original PDG, a two-playerstwo-strategies game, each player chooses one of the two available strategies, cooperation, or defection. Adopting the method of Yang's article [28], assuming that each piece of information or knowledge has equal value or utility and that any player has some knowledge that others have not, we can simplify the payoff matrix in accordance with common practice: a cooperator receives $R+S$ when playing with a cooperator and $-L$ when playing with a defector, while a defector earns 0 when playing with a defector and $R$ against a cooperator (as shown in Table 1). Here the effect of sharing knowledge consists of three components $R, S$, and $L$ :

(1) the basic value of information and knowledge for receivers, denoted by $R$, where $R \geq 0$;
TABLE 1: Basic payoff matrix.

\begin{tabular}{cccc}
\hline & & Player B \\
& & Cooperate & Betray \\
\hline \multirow{2}{*}{ Player A } & Cooperate & $R+S-L, R+S-L$ & $-L, R$ \\
& Betray & $R,-L$ & 0,0 \\
\hline
\end{tabular}

(2) the synergetic value describing the degree to which each agent gains because of the mutual information and knowledge transfer or sharing, represented by $S$, where $S \geq 0$;

(3) the perceived utility loss describing the degree to which each agent perceives the negative utility from the information and knowledge sharing activity due to the transfer of monopolistic knowledge, denoted by $-L ; L \geq 0$.

2.2. Three Situations of Innovation in Cooperation. After Schumpeter, according to whom technical knowledge is acquired both through invention and innovation [31], economists identified several kinds of innovations within technical change [32-34] according to the differentiation of innovation strength, mainly classified as incremental innovation and radical innovation [35]. And not all cooperation can give rise to innovation in the cooperation. So here we consider three types of situations which are incremental innovation, radical innovation, and no innovation.

Since we have different values for the parameters, we can have different results. But we just want to get the relationship between the behavior of cooperation and the development of the cluster, the evolution trend of the strategies, and the effects of incentive of polices. The size of the parameter selection does not have much of an impact on our simulation results; we pay attention to the relative size of the parameter selection. So we consider three types of payoff matrix.

(1) Situation I (no innovation): $R=5, L=2$, and $S=0$. In other words, first assume no synergy effect on Payoff matrix.

(2) Situation II (incremental innovation): $R=5, L=2$, and $S=2$. Assume a little synergy effect on innovation, producing the incremental effect on innovation.

(3) Situation III (radical innovation): $R=5, L=2$, and $S=5$. That is, the knowledge sharing might spark some new knowledge, producing the radical effect on innovation.

2.3. The Capability of Sharing and Absorbing Knowledge. When firms cooperate to exchange and share knowledge or resources, if not always explicit, the ability to exploit external knowledge is also a critical component of innovative capabilities $[36,37]$. On the bases of industry life of industrial cluster [38], the cluster developments were divided into three stages: introductory stage, growth stage, and maturity stage. In the early stage of the development of industry clusters, the firms' absorption capacity is weak. With the knowledge absorption, technology innovations continue to occur and 
TABLE 2: The payoff matrix with the different firms' capabilities.

\begin{tabular}{|c|c|c|c|}
\hline & & Player B & \\
\hline & & Cooperate & Betray \\
\hline Player A & Cooperate & $\begin{aligned} R * C_{S_{B}} * C_{a_{A}}+ & \left(C_{S_{A}} * C_{a_{A}}+C_{S_{B}} * C_{a_{B}}\right) * S / 2 ; \\
& -L * C_{S_{A}} * C_{a_{B}} \\
R * C_{S_{A}} * C_{a_{B}} & +\left(C_{S_{A}} * C_{a_{A}}+C_{S_{B}} * C_{a_{B}}\right) * S / 2 \\
& -L * C_{S_{B}} * C_{a_{A}}\end{aligned}$ & $\begin{array}{r}-L * C_{S_{A}} * C_{a_{B}} ; \\
R * C_{S_{A}} * C_{a_{B}}\end{array}$ \\
\hline & Betray & $R * C_{S_{B}} * C_{a_{A}} ;-L * C_{S_{B}} * C_{a A}$ & $0 ; 0$ \\
\hline
\end{tabular}

the firms' absorption capacity is growing [18]. Capabilities of firms in the cluster were used as probability distribution functions; three types of firms' capabilities were investigated in the stage of development of cluster.

(1) Stage I (stupid distribution): Stage I is introductory stage. The capabilities of the cluster members would be assumed as Beta probability distribution with $\alpha=$ 2 and $\beta=4$. It is a right-skewed distribution with mainly stupid members.

(2) Stage II (normal distribution): Stage II is growth stage. The capabilities of the cluster members is assumed to be a normal probability distribution with $\mu$ (mean) $=$ 0.5 and $\sigma^{2}$ (variance) $=0.16$.

(3) Stage III (smart distribution): Stage III is maturity stage. The capabilities of the cluster members have a Beta probability distribution with $\alpha=5$ and $\beta=$ 2. It is a left-skewed distribution with mainly smart members.

The firms' capabilities of knowledge sharing $\left(C_{S}\right)$ and absorption $\left(C_{a}\right)$ in different stages of the development of the cluster obey the different distribution which affect the firm's payoff of cooperation and might influence firm's behaviors. We assume the capabilities of firms affect $R$ and $L$ and the synergy effect of $S$ is affected by the mean of both firms' capabilities in the cooperation. So the payoff matrix is seen in Table 2.

2.4. The Incentive Policies. As is shown in Table 3, the payoff matrix is calculated given that a firm would be reward a positive incentive, denoted by I. The following three incentive policies were designed to explore the effectiveness of incentive policies on cooperation in the long terms. The degrees of incentives are implicitly degrees of risk premium for knowledge sharing by actors:

(1) no rewards: no incentive is provided for firm's cooperation;

(2) incentive I: rewards to each action of firm's cooperation. If a firm cooperates, it obtains a reward immediately. The reward is partly compensated for a firm's perceived loss due to sharing technology and knowledge with others, based on an algorithm that randomly assigns a floating-point number between 0 and 2 (depicted as $[0,2])$;
(3) incentive II: periodic rewards, if the ratio of sharing knowledge actions of an individual exceeds 50\% within three periods, then the firm is rewarded. Assume that the firm gets partly compensates which is the mean of a floating-point number between 0 and 2 (depicted as $[0,2])$.

2.5. Firms' Strategies. In the simulation, an agent adopts a strategy, which includes the rules responding to the opponent behaviors. Referring to the literature [28-30], four strategies (named uncooperative, cooperative, mistrustful, and tit-fortat in the following) were adopted in this experiment to investigate the effects on the agent behavior and performance of cluster cooperation frequencies:

(1) uncooperative: firms never share their knowledge with their opponents, irrespective of the actions adopted by opponents;

(2) cooperative: firms always share their knowledge with their opponents, regardless of the actions adopted by the opponents;

(3) mistrustful: firms initially do not share their knowledge with their opponents and later follow the action of opponents;

(4) tit-for-tat: firms initially share their knowledge with their opponents and later follow the action of their opponents.

Assuming that each agent has a memory of only one period memory, that is, it can only remember its opponent's behavior in the previous interaction, the combination of four strategies were applied to simulate the tendency of mutual trus tamong firms.

2.6. Mechanism of Learning and Adaptation. Given that all agents could observe their opponents' strategies and average payoff after a period of time, agents might adapt their strategies by comparing the average payoffs with their opponents. If one agent gains the largest average payoff, then other agents might, but not necessarily, with the assigned probability $20 \%$, learn the winner's strategy. Agents would imitate the best-performing strategy among their opponents in the experiment. 
TABLE 3: The payoff matrix with the different firms' capabilities while offering incentive.

\begin{tabular}{|c|c|c|c|}
\hline \multicolumn{4}{|c|}{ Player B } \\
\hline & & Cooperate & Betray \\
\hline \multirow[t]{2}{*}{ Player A } & Cooperate & $\begin{array}{c}R * C_{S_{B}} * C_{a_{A}}+\left(C_{S_{A}} * C_{a_{A}}+C_{S_{B}} * C_{a_{B}}\right) * S / 2 ; \\
-L * C_{S_{A}} * C_{a_{B}}+I \\
R * C_{S_{A}} * C_{a_{B}}+\left(C_{S_{A}} * C_{a_{A}}+C_{S_{B}} * C_{a_{B}}\right) * S / 2 \\
-L * C_{S_{B}} * C_{a_{A}}+I\end{array}$ & $\begin{array}{l}-L * C_{S_{A}} * C_{a_{B}}+I \\
\quad R * C_{S_{A}} * C_{a_{B}}\end{array}$ \\
\hline & Betray & $R * C_{S_{B}} * C_{a_{A}} ;-L * C_{S_{B}} * C_{a_{A}}+I$ & $0 ; 0$ \\
\hline
\end{tabular}

\section{Experimental Results}

All the simulations below are carried out on BA scale-free network with network size $N=400$ and $m 0=m=3$. The BA scale-free network which is generated by the rules of "growth" and "preferential attachment": starting from $m 0$ fully connected nodes, at every step a new node is added with $m$ edges that link to $m$ different existing nodes in such a way that the probability of being connected to the existing node $i$ is proportional to its degree.

Initially, different strategies are randomly distributed among the firms. The simulations consider three situations of innovation, the combination of behavioral strategies, capabilities in different stages, and incentive policies. Each parameter configuration was simulated repeatedly for 10 runs for taking up noises as possible, due to the differentiation of random exogenous parameters. In each run, the model was run for 100 periods and each agent would meet each opponent 4 times in each period. When interacting with other firms, the agent could recognize previous interactions, and remembered some aspects of previous outcomes. Firms would eventually adapt their own strategies for comparing average payoff with their opponents. The equilibrium frequencies of firms' behavior actions, strategies, and payoff were collected for each run. Finally, the average values of these variables were obtained for each parameter configuration.

We investigate the frequencies of cooperators' strategy in each situation of innovation and compare different incentives in the system.

3.1. The Evolvement of the Agents' Strategies in Situation I of Innovation. When cooperation between firms has no synergy effect on innovation, firms only share and absorb other cooperators' knowledge. Through simulation of the game theory, we can see average frequency of different strategy agents at the end of ten runs and the behavior of average value at the end of cooperation and noncooperation actions of ten runs.

As demonstrated in Figures 1(a)-1(c), when there are no incentives, firms in the cluster tend to tit-for-tat strategy in each stage of cluster development. Second is the cooperative strategy. The uncooperative strategy and mistrustful strategy account for only a small part. And an interesting observation was that the behavior of cooperation and the development of the cluster were $U$ shaped relationship. And the dominance of cooperative strategy is negative to the development of the cluster (shown in Table 4).
In Figures 1(d)-1(f), the firms in the cluster were partly compensated for the loss; due to sharing technology and knowledge with others, the compensation was a floatingpoint number between 0 and 2 . The cooperative strategy dominates other strategies in the cluster, followed by mistrustful strategy. The uncooperative strategy and mistrustful strategy account for only a small part also. But the behavior of cooperation becomes slightly worse with the development of the cluster (shown in Table 4).

In Figures 1(g)-1(i), when the ratio of sharing knowledge actions of a firm exceeds $50 \%$ within three periods, the firm gets partly compensation which is the mean of a floatingpoint number between 0 and 2 (depicted as $[0,2]$ ). With the development of the cluster the tit-for-tat strategy increasingly dominates other strategies in the cluster. The cooperative strategy is just the opposite. The uncooperative strategy and mistrustful strategy account for a small part which is a bit bigger than in the past. And the convergence speed is slowing down in this stage. The behavior of cooperation and the development of the cluster were inverted $U$ shaped relationship also (shown in Table 4).

Now an interesting observation was found; the incentive policy about compensating for each action increased the adoption of cooperative strategy and frequencies of cooperation in the cluster, even partly compensation for firms. But firms with the periodic fixed incentive policy could not suppress the number of agents with mistrustful and uncooperative strategies, even worse than no incentives. The reason for this can be showed by the convergence speed. Agents with the incentive for each action would easily change their initial strategy and attract other agents with worse performance to follow their behaviors. Hence, contrasting with the cases in which all players were with the incentive policy about compensating for each action, more cooperation occurred in these cases.

\subsection{The Evolvement of the Agents' Strategies in Situation II of} Innovation. This part analyzes the condition in which cooperation between firms can generate incremental innovation. Through simulation of the game theory, we can also see average frequency of different strategies and average value of cooperation and noncooperation actions.

As demonstrated in Figures 2(a)-2(c), when cooperation can generate incremental innovation, the cooperative strategy and tit-for-tat strategy dominate other strategies in the cluster. In the beginning development of the cluster, firms in the cluster tend to select tit-for-tat strategy more than 


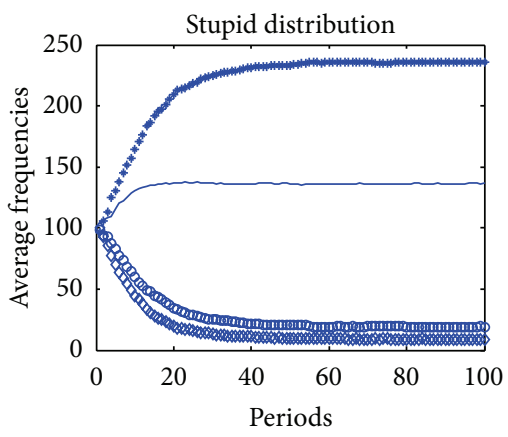

(a) No reward, stage I (introductory stage), situation I $(s=0)$

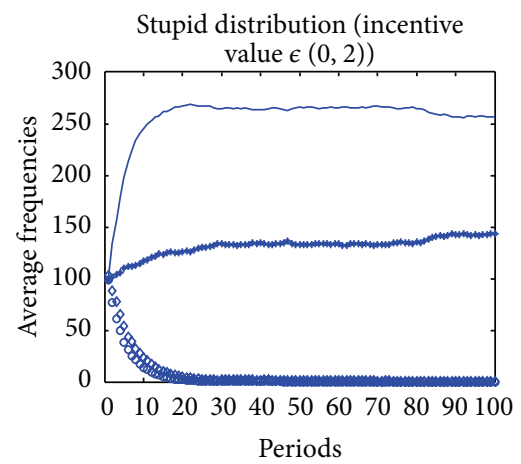

(d) Incentive I ([0, 2]), stage I (introductory stage), situation I $(s=0)$

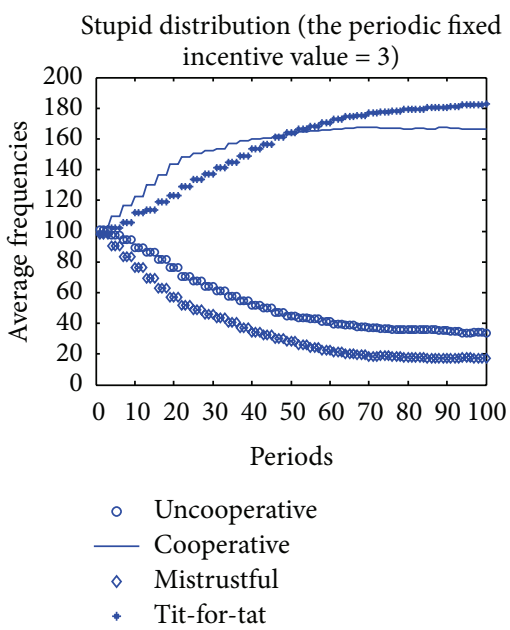

(g) Incentive II (3), stage I (introductory stage), situation I $(s=0)$

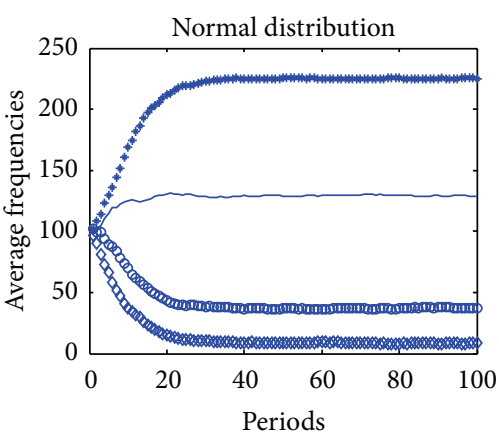

(b) No reward, stage II (growth stage), situation I $(s=0)$

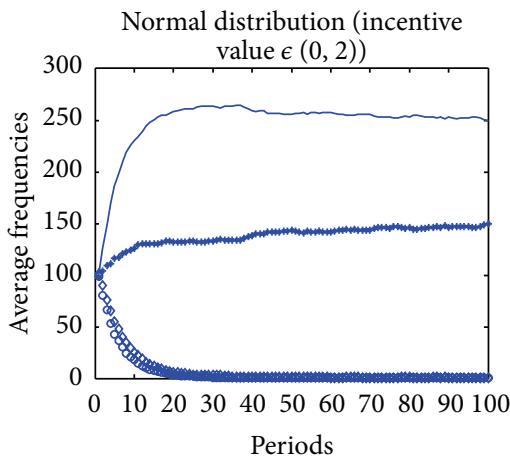

(e) Incentive I ([0, 2]), stage II (growth stage), situation I $(s=0)$

Normal distribution (the periodic fixed

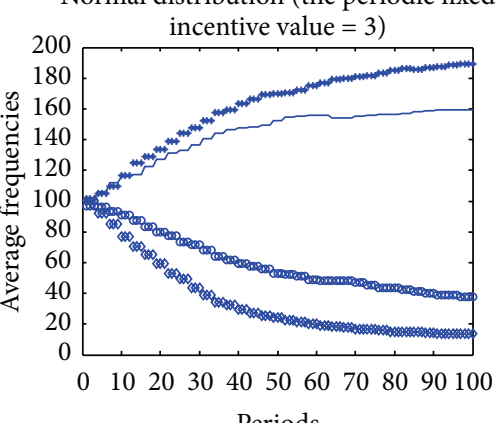

- Uncooperative

- Cooperative

$\diamond$ Mistrustful

- Tit-for-tat

(h) Incentive II (3), stage II (growth stage), situation I $(s=0)$

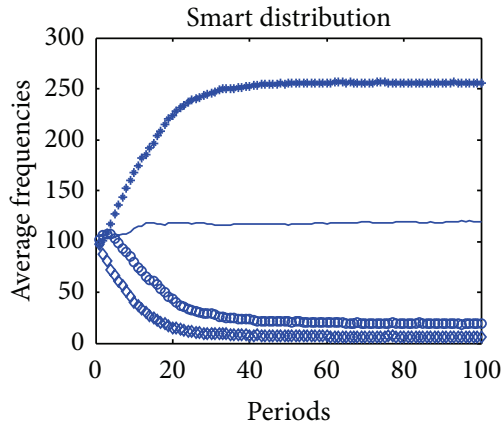

(c) No reward, stage III (maturity stage), situation I $(s=0)$

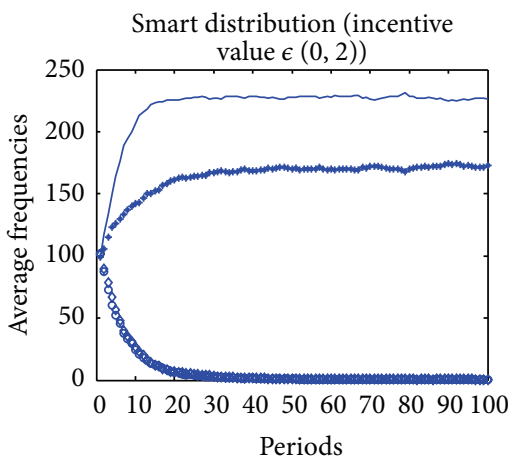

(f) Incentive I ([0, 2]), stage III (maturity stage), situation I $(s=0)$

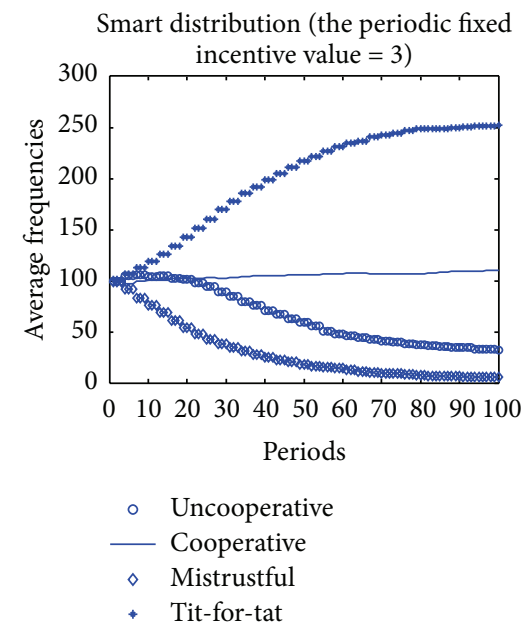

(i) Incentive II (3), stage III (maturity stage), situation I $(s=0)$

FIGURE 1: Dynamics about agents' strategy change in different stages in situation I of innovation.

cooperative strategy. But with the development of cluster the cooperative strategy increasingly dominates other strategies in the cluster. The uncooperative strategy and mistrustful strategy account for only a small part always. The behavior of cooperation becomes slightly better with the development of cluster (shown in Table 5).

In Figures 2(d)-2(f), the firms in the cluster were partly compensated for the loss. The compensation was a floating-point number between 0 and 2. The cooperative strategy dominates other strategies in the cluster, followed by mistrustful strategy. But with the development of cluster the number of tit-for-tat strategy increases and the gap is gradually narrowing. And the uncooperative strategy and mistrustful strategy have hardly any number. The behavior of cooperation becomes slightly better with the development of cluster (shown in Table 5).

In Figures 2(g)-2(i), when firms were compensated with periodic fixed incentive, the tit-for-tat strategy dominates 


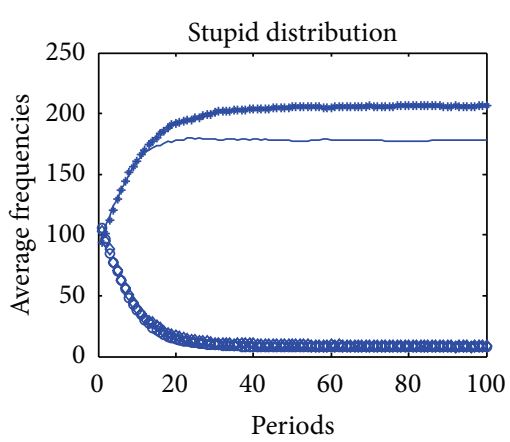

(a) No reward, stage I (introductory stage), situation II $(s=2)$

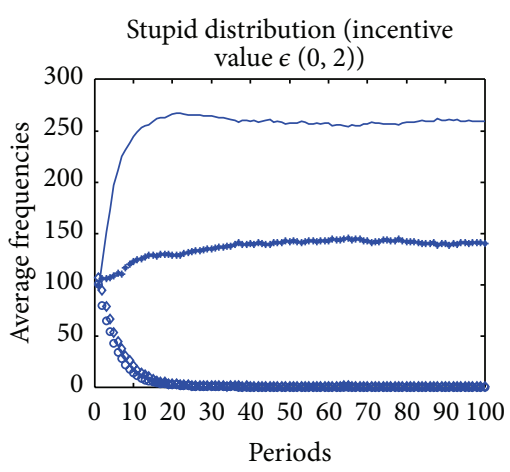

(d) Incentive I ([0, 2]), stage I (introductory stage), situation II $(s=2)$

Stupid distribution (the periodic fixed

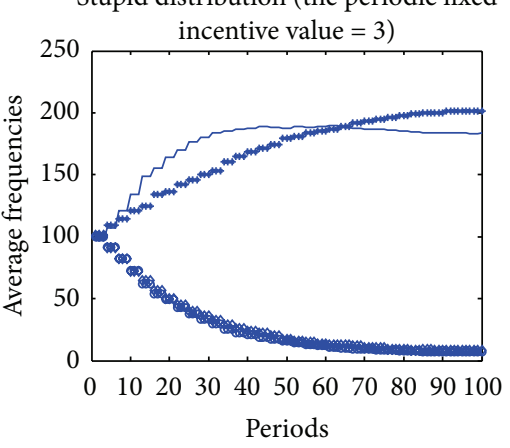

- Uncooperative

- Cooperative

$\diamond \quad$ Mistrustful

- Tit-for-tat

(g) Incentive II (3), stage I (introductory stage), situation II ( $s=2)$

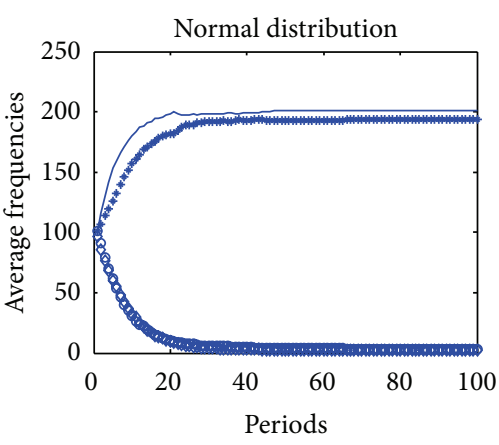

(b) No reward, stage II (growth stage), situation II $(s=2)$

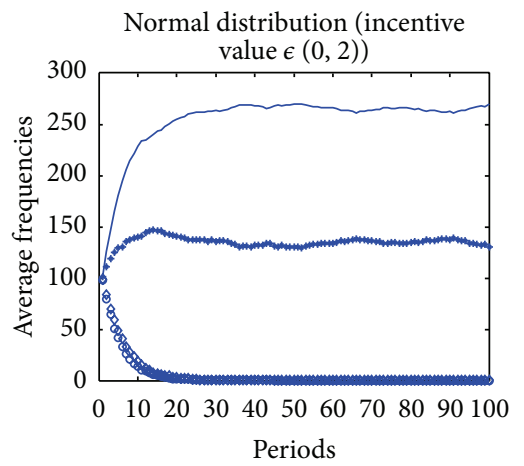

(e) Incentive I ([0, 2]), stage II (growth stage), situation II $(s=2)$

Normal distribution (the periodic fixed

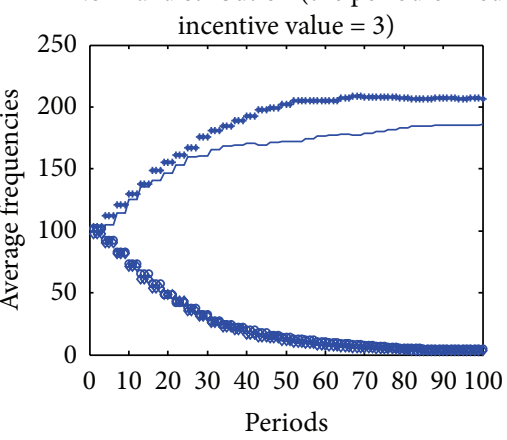

- Uncooperative

- Cooperative

$\diamond \quad$ Mistrustful

- Tit-for-tat

(h) Incentive II (3), stage II (growth stage), situation II $(s=2)$

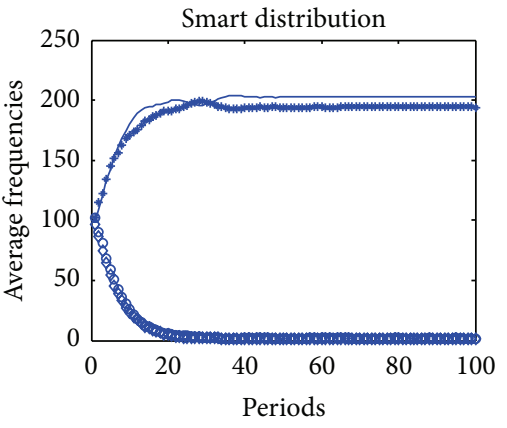

(c) No reward, stage III (maturity stage), situation II $(s=2)$

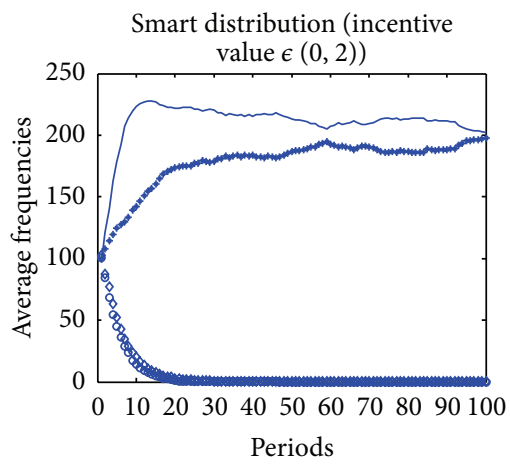

(f) Incentive I ([0, 2]), stage III (maturity stage), situation II $(s=2)$

Smart distribution (the periodic fixed

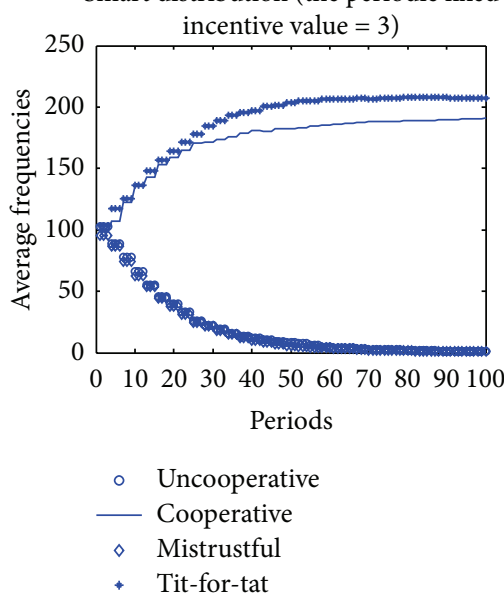

(i) Incentive II (3), stage III (maturity stage), situation II $(s=2)$

FIGURE 2: Dynamics about agents' strategy change in different stages in situation II of innovation.

other strategies in the cluster, followed by cooperative strategy. And with the development of cluster both of them increase (shown in Table 5). The uncooperative strategy and mistrustful strategy are just the opposite and account for only a small part. The behavior of cooperation becomes slightly better with the development of cluster (shown in Table 5).

Similarly, an interesting observation was that the incentive policy about compensating for each action increased the adoption of cooperative strategy and frequencies of cooperation in the cluster. But firms with the periodic fixed incentive policy could not suppress the number of agents with mistrustful and uncooperative strategies, and sometimes even worse than no incentives.

\subsection{The Evolvement of the Agents' Strategies in Situation III} of Innovation. This part analyzes the condition in which cooperation between firms can generate radical innovation. As demonstrated in Figures 3(a)-3(c), when there is no 


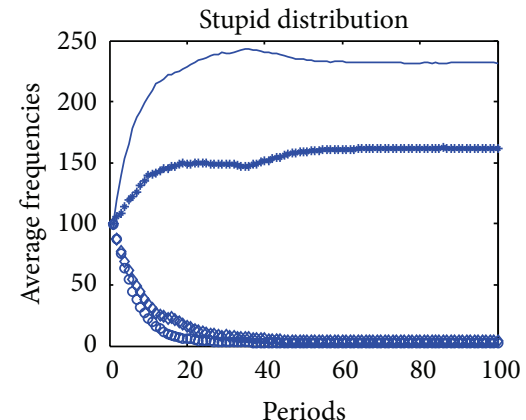

(a) No reward, stage I (introductory stage), situation III $(s=5)$

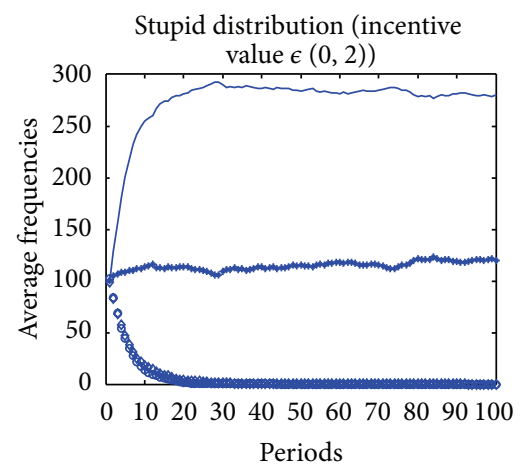

(d) Incentive I ([0, 2]), stage I (introductory stage), situation III $(s=5)$

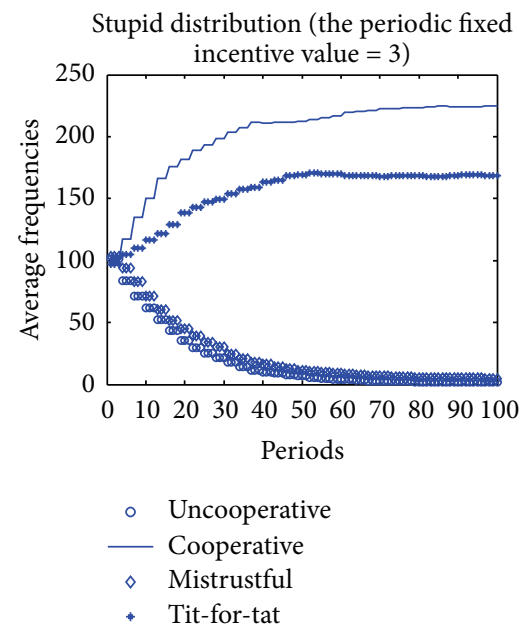

(g) Incentive II (3), stage I (introductory stage), situation III $(s=5)$

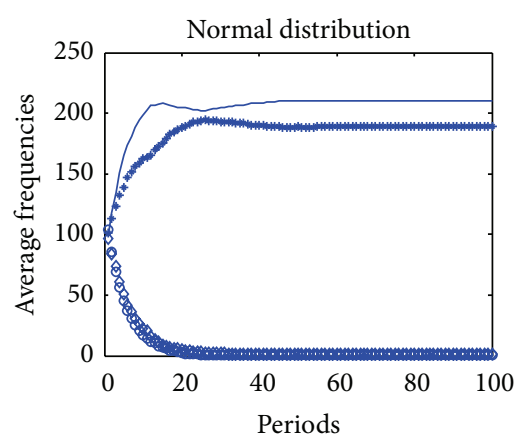

(b) No reward, stage II (growth stage), situation III $(s=5)$

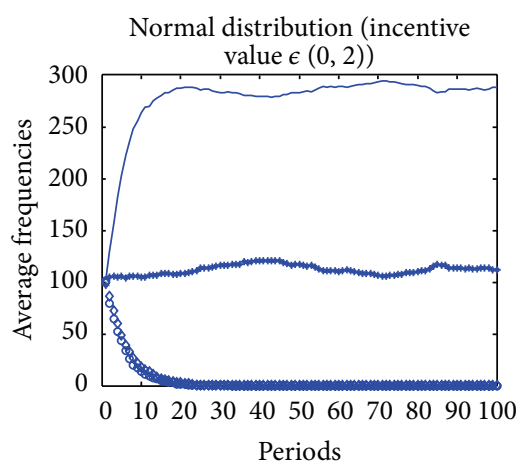

(e) Incentive I ([0, 2]), stage II (growth stage), situation III $(s=5)$

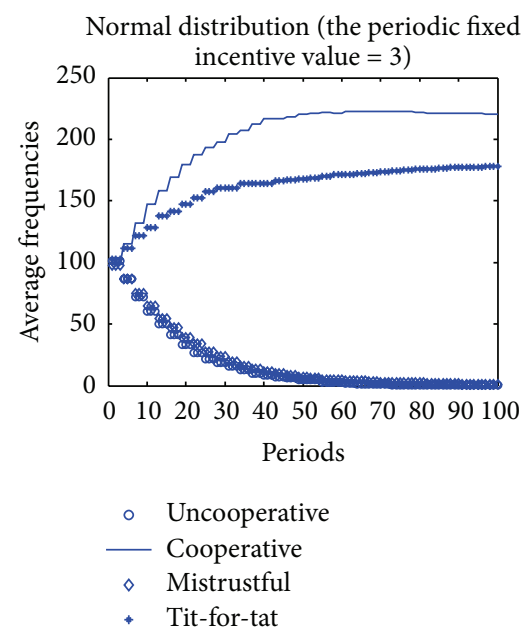

(h) Incentive II (3), stage II (growth stage), situation III $(s=5)$

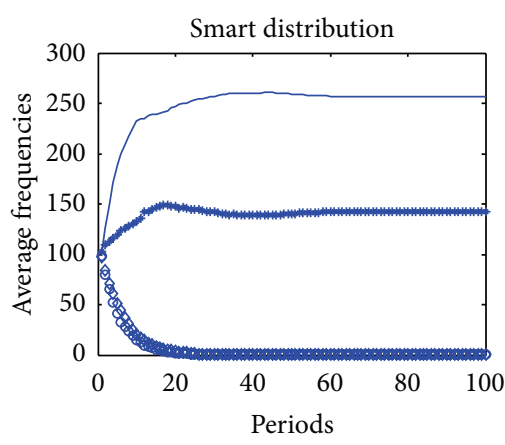

(c) No reward, stage III (maturity stage), situation III $(s=5)$

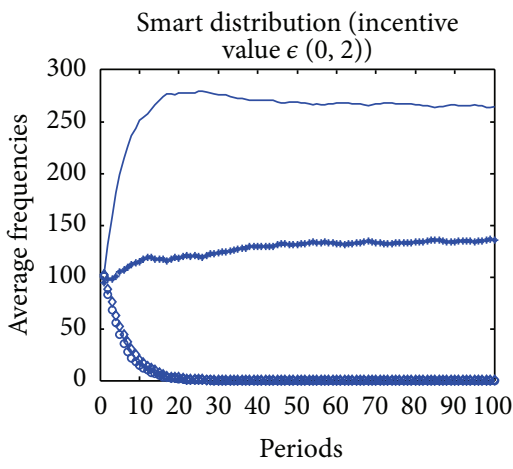

(f) Incentive I ([0, 2]), stage III (maturity stage), situation III $(s=5)$

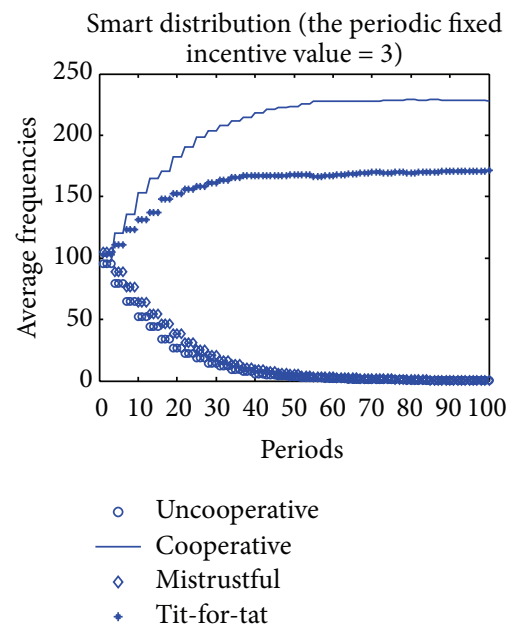

(i) Incentive II (3), stage III (maturity stage), situation III $(s=5)$

FIGURE 3: Dynamics about agents' strategy change in different stages in situation III of innovation.

incentive, the cooperative strategy dominates other strategies in the cluster, followed by tit-for-tat strategy. But the gap between them and the development of the cluster have an inverted U-shaped relationship. The uncooperative strategy and mistrustful strategy account for only a small part always three stages. The behavior of cooperation becomes better with the development of cluster (shown in Table 6).
In Figures 3(d)-3(f), the firms in the cluster were partly compensated for the loss; the compensation was a floatingpoint number between 0 and 2. The cooperative strategy dominates other strategies in the cluster, followed by tit-fortat strategy. And the uncooperative strategy and mistrustful strategy have hardly any number. This kind of situation has lasted for three stages. As to the behavior of cooperation, 
TABLE 4: Average frequencies of different strategy in Situation I of innovation with each incentive.

\begin{tabular}{|c|c|c|c|c|c|c|c|c|}
\hline \multirow{2}{*}{ Payoff } & \multirow{2}{*}{ Stage } & \multirow{2}{*}{ Reward } & \multicolumn{4}{|c|}{ Strategies } & \multicolumn{2}{|c|}{ Behavior } \\
\hline & & & Uncooperative & Cooperative & Mistrustful & Tit-for-tat & Cooperation & Noncooperation \\
\hline \multirow{9}{*}{ Situation I } & \multirow{3}{*}{ Stage I } & No reward & 18.9 & 136.8 & 8.8 & 235.5 & 8977.4 & 574.6 \\
\hline & & {$[0,2]$} & 0 & 256.3 & 0.4 & 143.3 & 9547.9 & 4.1 \\
\hline & & 3 & 33.8 & 166.1 & 17.3 & 182.8 & 8594.2 & 957.8 \\
\hline & \multirow{3}{*}{ Stage II } & No reward & 37.5 & 129 & 8.3 & 225.2 & 8712.5 & 839.5 \\
\hline & & {$[0,2]$} & 0.2 & 249.3 & 0.7 & 149.8 & 9544.5 & 7.5 \\
\hline & & 3 & 37.6 & 159.4 & 13.7 & 189.3 & 8494.2 & 1057.8 \\
\hline & \multirow{3}{*}{ Stage III } & No reward & 19.2 & 118.7 & 6.5 & 255.6 & 9008 & 544 \\
\hline & & {$[0,2]$} & 0.4 & 226.4 & 0.6 & 172.6 & 9536.4 & 15.6 \\
\hline & & 3 & 32.4 & 109.8 & 5.6 & 252.2 & 8702.3 & 849.7 \\
\hline
\end{tabular}

TABLE 5: Average frequencies of different strategy in Situation II of innovation with each incentive.

\begin{tabular}{|c|c|c|c|c|c|c|c|c|}
\hline \multirow{2}{*}{ Payoff } & \multirow{2}{*}{ Stage } & \multirow{2}{*}{ Reward } & \multicolumn{4}{|c|}{ Strategies } & \multicolumn{2}{|c|}{ Behavior } \\
\hline & & & Uncooperative & Cooperative & Mistrustful & Tit-for-tat & Cooperation & Noncooperation \\
\hline \multirow{9}{*}{ Situation II } & \multirow{3}{*}{ Stage I } & No reward & 7.6 & 177.7 & 8.6 & 206.1 & 9299.7 & 252.3 \\
\hline & & {$[0,2]$} & 0 & 259.5 & 0.2 & 140.3 & 9548.9 & 3.1 \\
\hline & & 3 & 6.7 & 183.7 & 8.2 & 201.4 & 9316 & 236 \\
\hline & \multirow{3}{*}{ Stage II } & No reward & 3.4 & 200.7 & 2.1 & 193.8 & 9467.1 & 84.9 \\
\hline & & {$[0,2]$} & 0 & 269.3 & 0.1 & 130.6 & 9551.1 & 0.9 \\
\hline & & 3 & 4.6 & 185.9 & 3.1 & 206.4 & 9413.9 & 138.1 \\
\hline & \multirow{3}{*}{ Stage III } & No reward & 1.7 & 203 & 1.2 & 194.1 & 9511.2 & 40.8 \\
\hline & & {$[0,2]$} & 0 & 202.1 & 0.1 & 197.8 & 9551.4 & 0.6 \\
\hline & & 3 & 0.9 & 190.9 & 1 & 207.2 & 9521.1 & 30.9 \\
\hline
\end{tabular}

nearly all the firms are willing to cooperate (shown in Table 6).

In Figures 3(g)-3(i), when firms were compensated with periodic fixed incentive, the distribution of different strategies is similar to the second case, as well as the behavior of cooperation (shown in Table 5).

The incentive policy about compensation for each action increased the adoption of cooperative strategy and frequencies of cooperation in the cluster, as well as the compensation with the periodic fixed incentive. But the policy with the periodic fixed incentive is a little worse than the policy with the compensation for each action.

3.4. The Evolvement of the Agents' Strategies in Different Situations of Innovation. When cooperation between firms was no incentive to compensate, the cooperative strategy and tit-for-tat strategy dominate other strategies in the cluster in each situation of innovation. As the payoff of the innovation is growing, the other two strategies are becoming more scarce and the cooperative strategy dominates other strategies in the cluster (Figures 1(a)-1(c), 2(a)-2(c), and 3(a)-3(c)). Also the behavior of cooperation becomes better (shown in Tables $4-$ 6).

When the firms in the cluster were partly compensated for the loss, the compensation was a floating-point number between 0 and 2 . The cooperative strategy dominates other strategies in the cluster, followed by mistrustful strategy
(Figures 1(d)-1(f), 2(d)-2(f), and 3(d)-3(f)). With the development of cluster the number of tit-for-tat strategy decreases and the behavior of cooperation becomes slightly better (shown in Tables 4-6).

Similar to the situation of no incentive policy, when the firms were compensated with periodic fixed incentive, the cooperative strategy and tit-for-tat strategy dominate other strategies in the cluster in each type of innovation. As the payoff of the innovation is growing, the other two strategies are becoming more scarce and the cooperative strategy dominates other strategies in the cluster (Figures $1(\mathrm{~g})-1(\mathrm{i})$, $2(\mathrm{~g})-2(\mathrm{i})$, and $3(\mathrm{~g})-3(\mathrm{i}))$. Also the behavior of cooperation becomes better (shown in Tables 4-6).

\section{Discussion and Conclusion}

At the beginning of this paper, we raised a question about the contribution of the innovation network structure and the heterogeneity of firms' capability to the behaviors of cooperation in the cluster. Previous researches mainly focus on the strategies' effect on cooperation or the network structure influence on the effects of tacit knowledge transfer. But the relationship between network structure and the strategies' effect on cooperation had not been examined directly. Also the different stage of cluster development and different incentive policies had not been considered. We focused on a typical network structure, the scale-free network, and examined the 
TABLE 6: Average frequencies of different strategy in Situation III of innovation with each incentive.

\begin{tabular}{|c|c|c|c|c|c|c|c|c|}
\hline \multirow{2}{*}{ Payoff } & \multirow{2}{*}{ Stage } & \multirow{2}{*}{ Reward } & \multicolumn{4}{|c|}{ Strategies } & \multicolumn{2}{|c|}{ Behavior } \\
\hline & & & Uncooperative & Cooperative & Mistrustful & Tit-for-tat & Cooperation & Noncooperation \\
\hline \multirow{9}{*}{ Situation III } & \multirow{3}{*}{ Stage I } & No reward & 2.4 & 231.6 & 4.2 & 161.8 & 9471.3 & 80.7 \\
\hline & & {$[0,2]$} & 0 & 280.2 & 0 & 119.8 & 9552 & 0 \\
\hline & & 3 & 1.5 & 224.9 & 4.9 & 168.7 & 9481.9 & 70.1 \\
\hline & \multirow{3}{*}{ Stage II } & No reward & 0 & 278.9 & 0.2 & 120.9 & 9551.4 & 0.6 \\
\hline & & {$[0,2]$} & 0 & 287.8 & 0.1 & 112.1 & 9551.4 & 0.6 \\
\hline & & 3 & 0.2 & 220.5 & 1.2 & 178.1 & 9541.4 & 10.6 \\
\hline & \multirow{3}{*}{ Stage III } & No reward & 0.3 & 256.7 & 0.3 & 142.7 & 9544.6 & 7.4 \\
\hline & & {$[0,2]$} & 0 & 264.2 & 0.1 & 135.7 & 9551.4 & 0.6 \\
\hline & & 3 & 0.2 & 227.9 & 0.5 & 171.4 & 9546.1 & 5.9 \\
\hline
\end{tabular}

strategies' effect on cooperation in this network. The evidence indicated that network structure influence the behaviors of cooperation, compared with Yang's article. The findings are important because they clarify and extend past research.

The findings clarify and extend our understanding of the theory of industrial cluster. Previous research has emphasized the importance of different network structures effects on the transfer of codified knowledge in the cluster. We test the scalefree network structure influence on the behaviors of cooperation in different development stages of industrial cluster. With the development of industry cluster, the strategies adopted by firms are constantly evolving. But different industry cluster has different network structure, so the evolvement of strategies is different. And the development stages of industrial clusters with different network structure also affect the behavior of cooperation. This paper has only studied the typical scale-free network structure. So to understand the relationship between the network structure and the behavior of cooperation is meaningful.

The findings also provide some insight into how incentive policies can affect the behavior of cooperation. If we wish to encourage firms to cooperate and share their knowledge, then the best incentive policy is to reward each action of knowledge sharing. Because cooperative strategy dominated other strategies, the climate will be more trustworthy in cluster. The incentive policies influence on the behavior of cooperation will be affected by industrial clusters with different network structure, also with different development stages.

To conclude, our paper differentiates three situations of innovation and simulates the actions of cooperation between firms with different life cycles in industry cluster. Considering the real situation of the industrial cluster network, there is a very important network structure, the scale-free network. The simulations produce the following interesting findings. (1) When the industry cluster develops, firms' absorbability also is growing, but the behavior of cooperation does not always increase as firms' absorbability grows. Sometimes they exhibit inverted U-shape, U-shape, or liner trend. (2) Firms will cooperate to share their information or knowledge automatically if the payoff of the innovation is bigger. (3) The policy of compensation for each cooperation action is more effective than the policy of compensation for periodic fixed incentive.

However, this study still has certain limitations. The setup of our model is comparatively simple while that of the cooperation between firms in the cluster for innovation in real life is much more complex. Therefore, the priority of our future study will focus on a model whose setup is even closer to the one in real life, which could include considering the products competition, ego satisfaction, the motivations of sharing knowledge, and so forth. And also some real network structure will be studied by some survey method, since the network structure is really important to the evolution process.

\section{Conflict of Interests}

The authors declare that there is no conflict of interests regarding the publication of this paper.

\section{Acknowledgments}

This research was supported by NSFC (nos. 71273047 and 71172044) and the Major Program of the National Social Science Foundation of China (Grant no. 12\&ZD207).

\section{References}

[1] R. Gulati, "Does familiarity breed trust? The implications of repeated ties for contractual choice in alliances," Academy of Managemnet Journal, vol. 38, no. 1, pp. 85-112, 1995.

[2] R. Gulati, "Social structure and alliance formation patterns: a longitudinal analysis," Administrative Science Quarterly, vol. 40, no. 4, pp. 619-652, 1995.

[3] J. Hagedoorn, New Explorations in the Economics of Technical Change, 1990.

[4] D. J. Teece, "Competition, cooperation, and innovation. Organizational arrangements for regimes of rapid technological progress," Journal of Economic Behavior and Organization, vol. 18, no. 1, pp. 1-25, 1992.

[5] W. W. Powell, K. W. Koput, and L. Smith-Doerr, "Interorganizational collaboration and the locus of innovation: networks of learning in biotechnology," Administrative Science Quarterly, vol. 41, no. 1, pp. 116-145, 1996. 
[6] Jaffe, A. B, Trajtenberg, M, Henderson, and R, "Geographic localization of knowledge spillovers as evidenced by patent citations," Quarterly Journal of Economics, vol. 108, no. 3, pp. 577-598, 1993.

[7] S. Barrios, L. Bertinelli, and E. Strobl, "Coagglomeration and spillovers," Regional Science and Urban Economics, vol. 36, no. 4, pp. 467-481, 2006.

[8] M. Fujita and J. F. Thisse, Economics of Agglomeration. Cities, Industrial Location and Regional Growth, Cambridge University Press, Cambridge, UK, 2002.

[9] J. Bessant, A. Alexander, G. Tsekouras, H. Rush, and R. Lamming, "Developing innovation capability through learning networks," Journal of Economic Geography, vol. 12, no. 5, pp. 1087-1112, 2012.

[10] K. J. Arrow, "The economic implications of learning bydoing," The Review of Economic Studies, vol. 29, no. 3, pp. 155-173, 1962.

[11] P. M. Romer, "Increasing returns and long-run growth," Journal of Political Economy, vol. 94, no. 5, pp. 1002-1037, 1986.

[12] M. E. Porter, "Clusters and the new economics of competition," Harvard Business Review, vol. 76, no. 6, pp. 77-90, 1998.

[13] M. E. Porter and S. Stern, "Innovation: Location Matters," MIT Sloan Management Review, vol. 42, no. 4, pp. 28-36, 2001.

[14] J. Koo, “Technology spillovers, agglomeration, and regional economic development," Journal of Planning Literature, vol. 20, no. 2, pp. 99-115, 2005.

[15] A.-L. Barabási and R. Albert, "Emergence of scaling in random networks," Science, vol. 286, no. 5439, pp. 509-512, 1999.

[16] B. Uzzi, "Social structure and competition in interfirm networks: the paradox of embeddedness," Administrative Science Quarterly, vol. 42, no. 1, pp. 35-67, 1997.

[17] J. A. C. Baum, R. Cowan, and N. Jonard, "Network-independent partner selection and the evolution of innovation networks," Management Science, vol. 56, no. 11, pp. 2094-2110, 2010.

[18] R. Cowan, N. Jonard, and M. Özman, "Knowledge dynamics in a network industry," Technological Forecasting and Social Change, vol. 71, no. 5, pp. 469-484, 2004.

[19] R. Cowan and N. Jonard, "Network structure and the diffusion of knowledge," Journal of Economic Dynamics \& Control, vol. 28, no. 8, pp. 1557-1575, 2004.

[20] X. Yang and L. X. Yang, “Towards the epidemiological modeling of computer viruses," Discrete Dynamics in Nature and Society, vol. 2012, Article ID 259671, 11 pages, 2012.

[21] C. Fang, J. Lee, and M. A. Schilling, "Balancing exploration and exploitation through structural design: the isolation of subgroups and organizational learning," Organization Science, vol. 21, no. 3, pp. 625-642, 2010.

[22] F. Fu, X. Chen, L. Liu, and L. Wang, "Social dilemmas in an online social network: the structure and evolution of cooperation," Physics Letters A: General, Atomic and Solid State Physics, vol. 371, no. 1-2, pp. 58-64, 2007.

[23] J. Poncela, J. Gómez-Gardeñes, L. M. Floría, and Y. Moreno, "Robustness of cooperation in the evolutionary prisoner's dilemma on complex networks," New Journal of Physics, vol. 9, article 184, 2007.

[24] R. Axelrod, The Evolution of Cooperation, Basic Books, New York, NY, USA, 1984.

[25] J. Hofbauer and K. Sigmund, Evolutionary games and population dynamics, Cambridge University Press, Cambridge, 1998.

[26] W.-B. Du, X.-B. Cao, M.-B. Hu, H.-X. Yang, and H. Zhou, "Effects of expectation and noise on evolutionary games,"
Physica A: Statistical Mechanics and its Applications, vol. 388, no. 11, pp. 2215-2220, 2009.

[27] G. Szabó and G. Fáth, "Evolutionary games on graphs," Physics Reports, vol. 446, no. 4-6, pp. 97-216, 2007.

[28] H.-L. Yang and T. C. T. Wu, "Knowledge sharing in an organization," Technological Forecasting and Social Change, vol. 75, no. 8, pp. 1128-1156, 2008.

[29] M. A. Nowak and K. Sigmund, "Tit for tat in heterogeneous populations," Nature, vol. 355, no. 6357, pp. 250-253, 1992.

[30] M. Nowak and K. Sigmund, "A strategy of win-stay, lose-shift that outperforms tit-for-tat in the Prisoner's Dilemma game," Nature, vol. 364, pp. 56-58, 1993.

[31] J. Schumpeter, Business Cycles, McGraw-Hill, New York, NY, USA, 1939.

[32] T. Durand, "Dual technological trees: assessing the intensity and strategic significance of technological change," Research Policy, vol. 21, no. 4, pp. 361-380, 1992.

[33] K. B. Clark, "The interaction of design hierarchies and market concepts in technological evolution," Research Policy, vol. 14, no. 5, pp. 235-251, 1985.

[34] C. Freeman, "The greening of technology and models of innovation," Technological Forecasting and Social Change, vol. 53, no. 1, pp. 27-39, 1996.

[35] E. Mansfield, Industrial Research and Technological Innovation, Norton, New York, NY, USA, 1968.

[36] R. Gulati, N. Nohria, and A. Zaheer, "Strategic networks," Strategic Management Journal, vol. 21, no. 3, pp. 203-215, 2000.

[37] W. M. Cohen and D. A. Levinthal, "Absorptive capacity: a new perspective on learning and innovation," Administrative Science Quarterly, vol. 35, no. 1, pp. 128-152, 1990.

[38] E. V. Karniouchinal, S. J. Carson, J. C. Short, and D. J. Ketchen, "Extending the firm versus industry debate: does industry life cycle stage matter?" Strategic Management Journal, vol. 34, no. 8, pp. 1010-1018, 2013. 


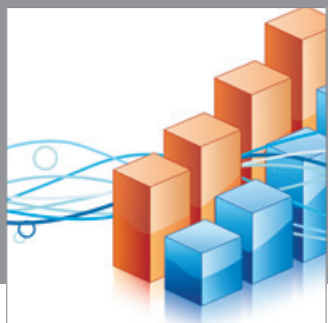

Advances in

Operations Research

mansans

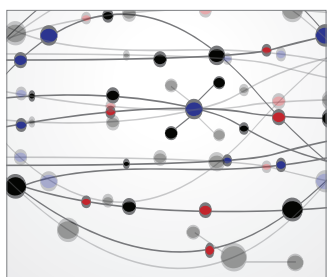

The Scientific World Journal
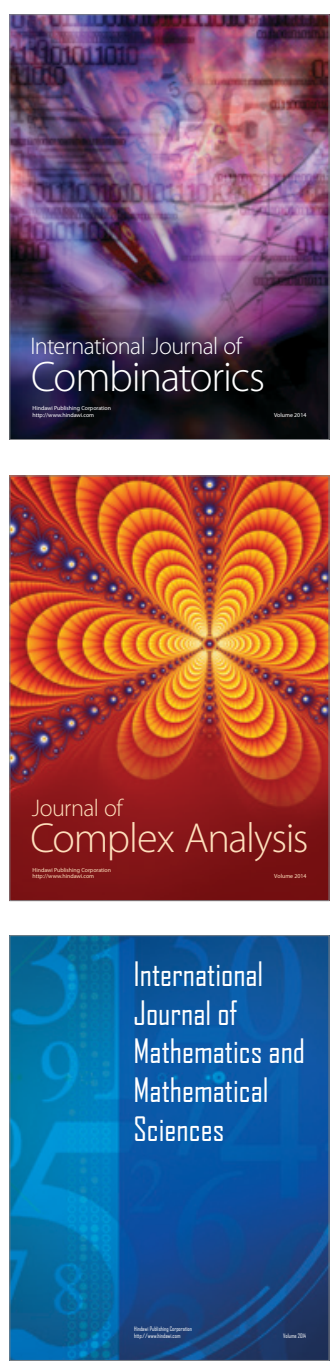
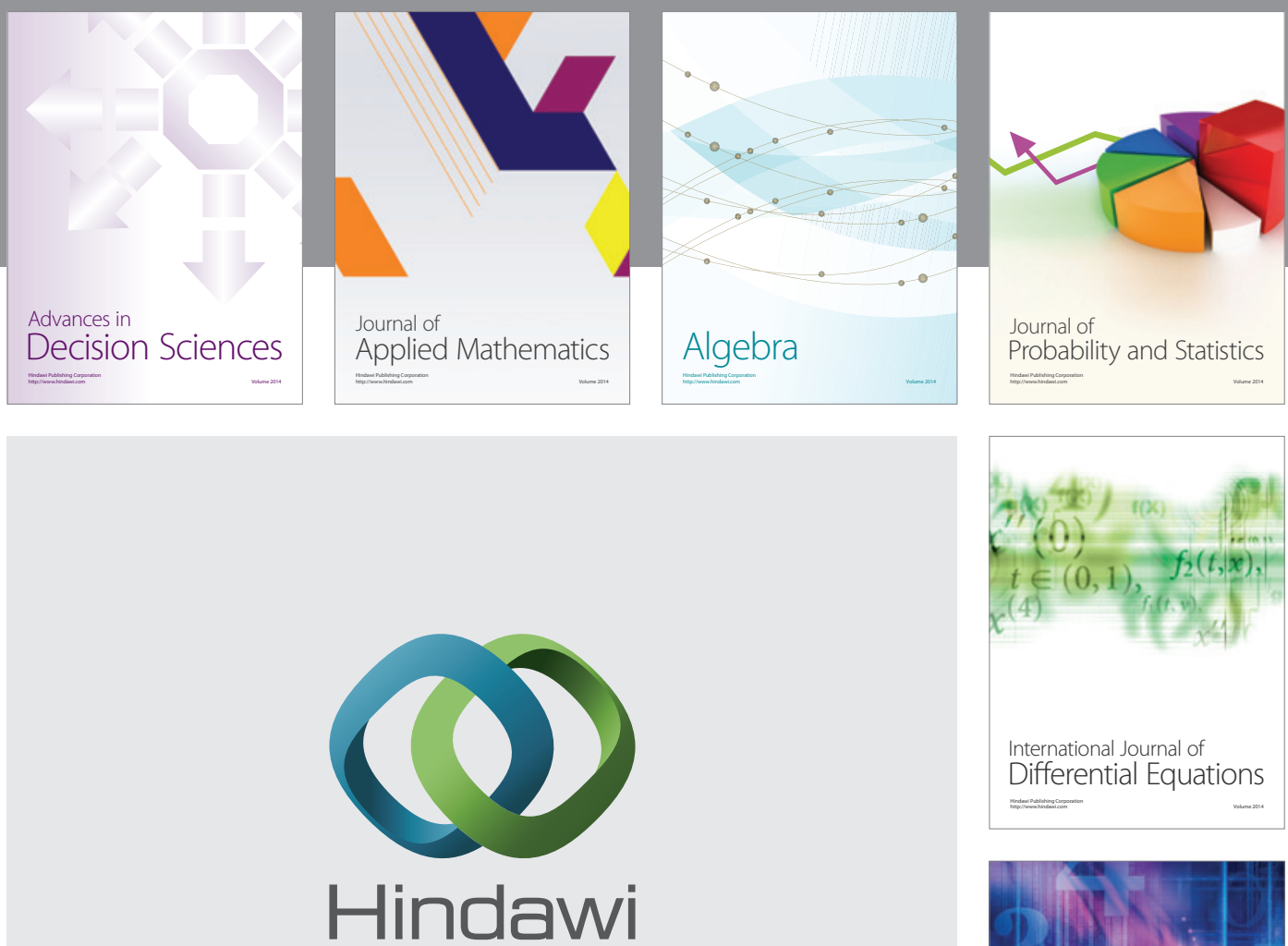

Submit your manuscripts at http://www.hindawi.com
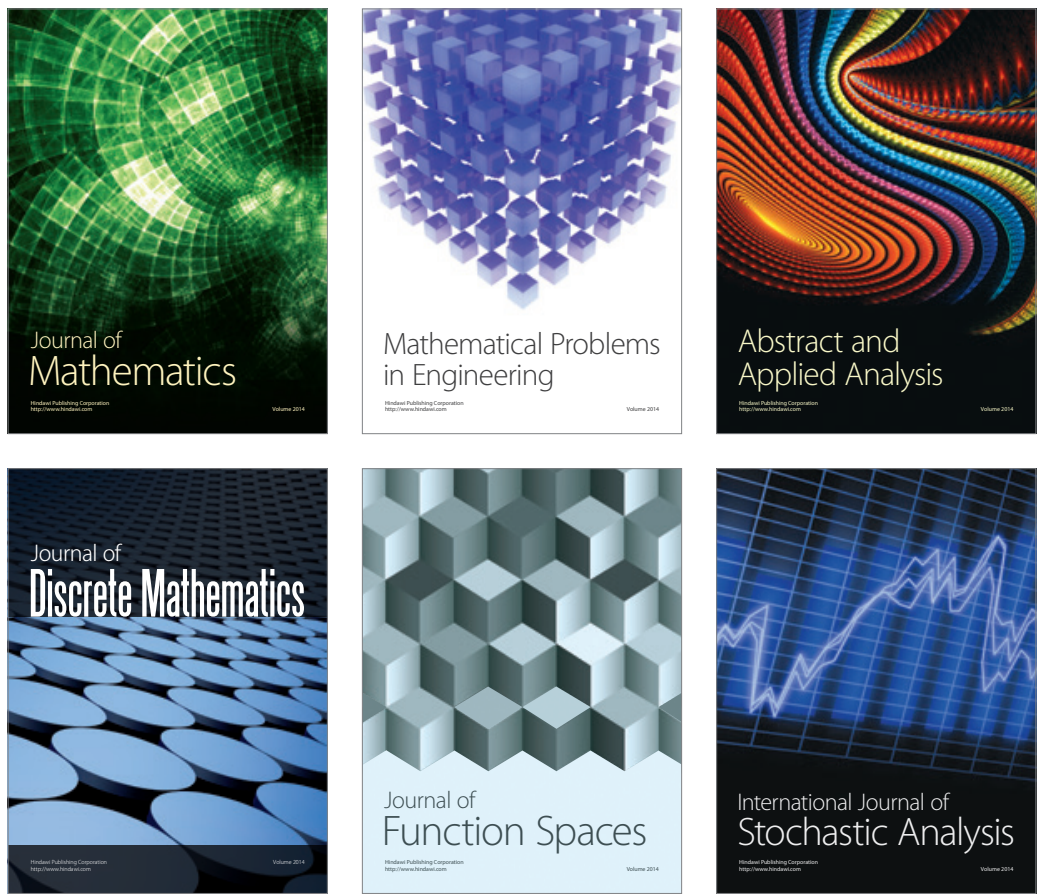

Journal of

Function Spaces

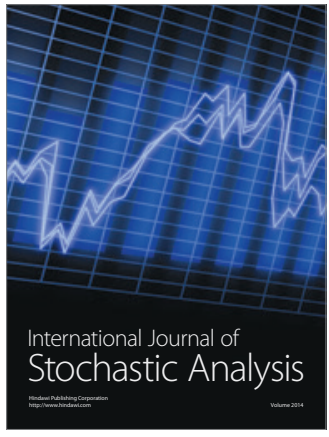

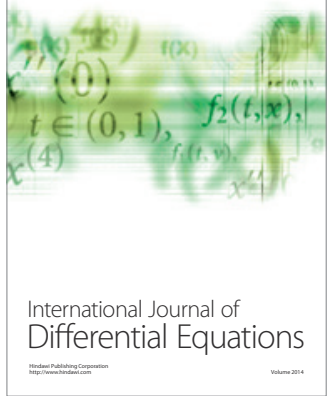
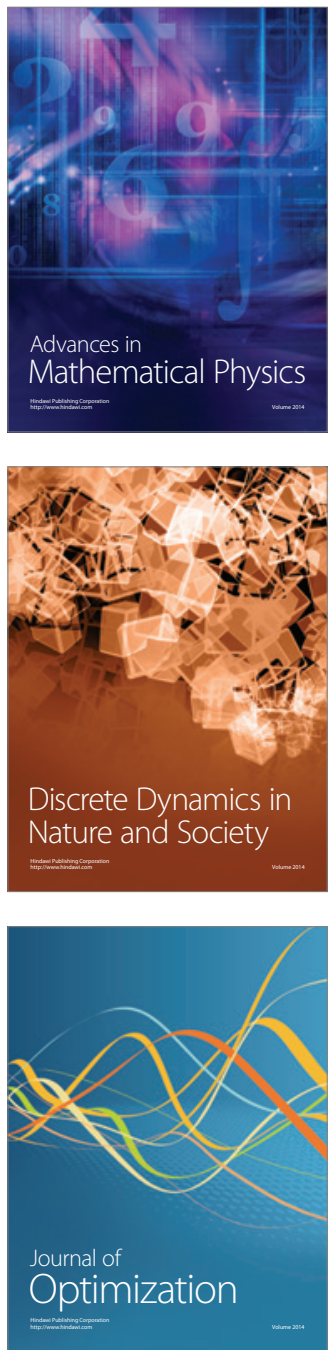